\title{
Study on the Mechanical Properties of Grouting-sleeve Reinforcement- connection Component under the Action of Cyclic Tension Load
}

\author{
Fan $\mathrm{Gu}^{1}{ }^{\text {, a }}$, Wei Wei ${ }^{1}$, Weijian Zhao ${ }^{1}$, Aike Xian ${ }^{1}$ \\ ${ }^{1}$ Civil Engineering College, Shenyang Jianzhu University, China \\ aguzhaozheng@yeah.net
}

\begin{abstract}
Keywords: Grouting-sleeve Reinforcement-connection Component; Cyclic Tension Load; Stress Distribution; Grouting-material; ABAQUS

Abstract. By means of finite element software ABAQUS, the numerical model of grouting-sleeve reinforcement-connection component under the action of axial load was established. Two kinds of loading system were considered, one was axial tension load with constant loading rate, and another was unidirectional cyclic load. The latter loading system simulates frequent earthquake action. The stress distribution of grouting-sleeve reinforcement-connection component in accordance with two kinds of loading system was calculated, as well as the compressive stress variation of grouting-material was studied, which provides the reference for further research on the mechanical properties of grouting-sleeve reinforcement-connection components.
\end{abstract}

\section{Introduction}

With the development of architectural industrialization, prefabricated construction has extensive promotion prospect. Compared with traditional reinforced concrete structure, prefabricated structure has advantages of convenient construction and reliable performance [1]. Developed countries began research and practice it since the middle of the 20th century and acquired abundant technological experience. In the process of prefabricated construction, prefabricated parts such as beams and columns are manufactured in factory beforehand, and then are connected together by reinforcementconnection component at job site, which plays a decisive role.

Reinforcement-connection technology by grouting-sleeve is that two steel reinforcement bars are inserted into cast iron sleeve, and then grouting-material is injected into sleeve by filling equipment. As this kind of reinforcement-connection method having advantages such as high joint strength, reliable performance, energy saving, convenient operation and rapid construction, it has become the main way for reinforcement bars connection in prefabricated construction filed. In1994, Yang [2] studied and introduced grouting-sleeve reinforcement-connection technology from Japan. Wu [3] took research on seismic performance of grouting-sleeve reinforcement-connection component by means of static experiment. Nobuyuki [4] studied the overall mechanical property of prefabricated column adopting grouting-sleeve reinforcement-connection technology by static experiment, and put forward the influence of grouting-material strength on the mechanical behavior of components. Considering the material characteristics and production process in various stages of prefabricated construction, Heng Qin [5] analyzed the influencing factors on the connection quality of grouting- sleeve reinforcement-connection component, and put forward technological measures to guarantee prefabricated construction quality.

At present, scholars all over the world have carried out research on grouting-sleeve reinforcementconnection component in various aspects. However, there are few studies on the mechanical property of component under the action of unidirectional cyclic load, which simulates the case of earthquake when its intensity below the regional seismic fortification intensity. In this paper, the numerical model of component for connecting $\Phi 20 \mathrm{~mm}$ reinforcement was established, and the mechanical response of component under the action of static load and unidirectional cyclic load were analyzed respectively, which lay a foundation for the optimal design and popularization of component. 


\section{Numerical Simulation of Grouting-sleeve Reinforcement-connection Component}

On the basis of grouting-sleeve structure, the finite element numerical model of grouting-sleeve reinforcement-connection component for connecting $\Phi 20 \mathrm{~mm}$ reinforcement was established by ABAQUS. According to the symmetrical behavior of component in axial direction, the half model in axial direction was selected for reducing the total number of elements. Meanwhile, under the action of axial load, it can be regarded as axisymmetric problem. The mother surface of the axisymmetric components model is shown as Figure1, with the corresponding displacement boundary condition applied on the left end of sleeve and axial load acting on the left end of steel. In the process of building numerical model, non-distortion-linear reducing-integral axisymmetric four-node quadrilateral unit (CAX4R) was adopted for grid partition. The total number of element of model was up to 963, and the total number of node of model was up to 1219.

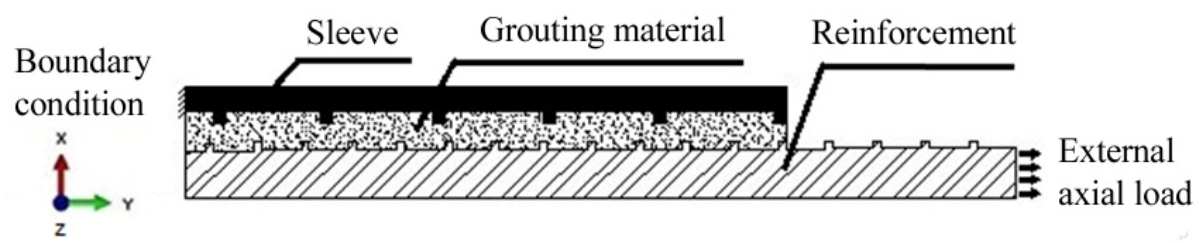

Fig. 1 The numerical model of grouting-sleeve reinforcement-connection component

During the process of establishing numerical model, the concrete damaged plasticity model was adopted for representing grouting-material constitutive relation. According to literature [6], stressstrain curve of grouting-material was obtained. Meanwhile, the constitutive data of reinforcement and cast iron with reference to literature [7] were adopted respectively.

For numerical model of grouting-sleeve reinforcement-connection component, two kinds of loading system were adopted as shown in Table1. One is termed as reference loading system, which means axial tensile load with constant loading rate $8 \mathrm{MPa} / \mathrm{s}$ is imposed on reinforcement until up to 0.9 times of steel yield strength $f_{\mathrm{yk}}$. Another is termed as experimental loading system, which means axial unidirectional cyclic load is imposed on reinforcement in accordance with standard inspection regulations JGJ107, quod vide Table1 in detail.

Table1 Loading system adopted for numerical model of component

\begin{tabular}{cc}
\hline Specimen number & Loading system \\
\hline $20 \mathrm{GS}-1$ & Load with constant loading rate of $8 \mathrm{MPa} / \mathrm{s} \rightarrow f_{\mathrm{yk}}$ \\
$20 \mathrm{GS}-2$ & Unidirectional cyclic load: $0 \rightarrow 0.6 f_{\mathrm{yk}} \rightarrow 0.02 f_{\mathrm{yk}} \rightarrow 0.6 f_{\mathrm{yk}} \rightarrow 0.02 f_{\mathrm{yk}} \rightarrow 0.6 f_{\mathrm{yk}}$ \\
\hline
\end{tabular}

Annotation : $f_{\mathrm{yk}}-$ standard value of steel yield strength.

\section{Results and Discussion}

For test specimen 20GS-1, axial tensile load with constant loading rate of $8 \mathrm{MPa} / \mathrm{s}$ was imposed on the end of reinforcement. When tensile load was up to $360 \mathrm{Mpa}$, which is 0.9 times of steel yield strength $f_{\mathrm{yk}}$, the Mises stress nephogram of component was gotten shown in Figure2(a). Meanwhile, the Mises stress distribution at reinforcement and cast iron sleeve are shown as Figure2(b) and (d) respectively. As grouting-material being in compressive status, the minimum-principal-stress distribution at grouting-material is presented as Figure2(c). From Figure2, it can be seen that the Mises stress at reinforcement bars decreases form loading end to free end in axial direction, whereas the Mises stress at sleeve decreases from mid-span cross section to free end cross section. In addition, the minimum-principal-stress at grouting-material is compressive, and its variation range is limited. 
Furthermore, between reinforcing rib and corresponding sleeve rib, lamp-umbrella compressive zone in grouting-material was formed for load transmission from reinforcement to sleeve.

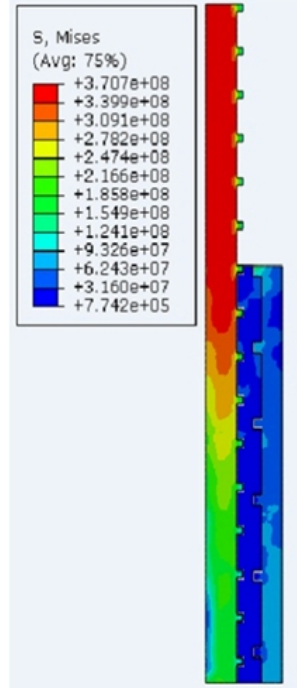

(a)

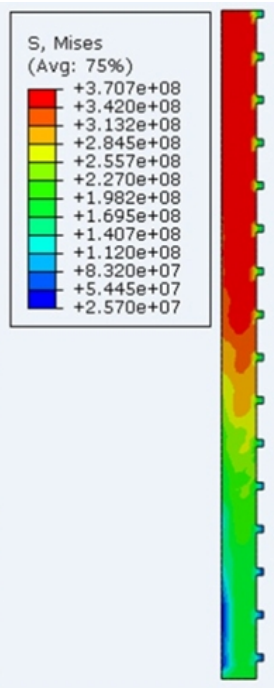

(b)

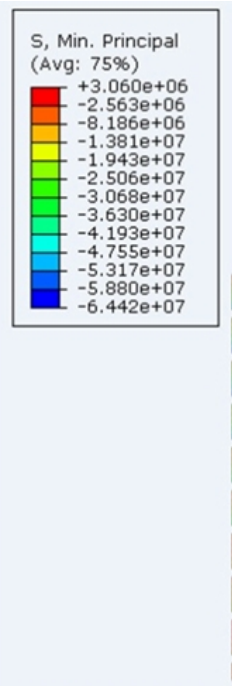

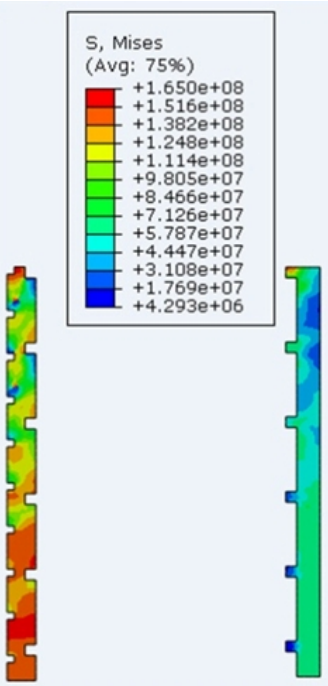

(c) (d)

Fig.2. The stress nephogram of 20GS-1 specimen component

For test specimen 20GS-2, unidirectional cyclic load was imposed on the end of reinforcement, and the final stress nephogram of component, reinforcement, grouting-material and sleeve are as shown in Figure3(a) - (d) respectively. Compared with Figure2, it can be seen that the stress variation trends of specimen 20GS-2 are similar to that of specimen 20GS-1. For test specimen 20GS-1 and 20GS-2, numerical simulation result shows that the maximum Mises stress of reinforcement locating at loading end is $370 \mathrm{MPa}$ and $244 \mathrm{MPa}$ respectively, which does not reach the yield strength of steel. Meanwhile, for test specimen 20GS-1 and 20GS-2, the maximum Mises stress of sleeve locating at mid-span cross section is $165 \mathrm{MPa}$ and $115 \mathrm{MPa}$ respectively, which does not reach the yield strength of cast iron.

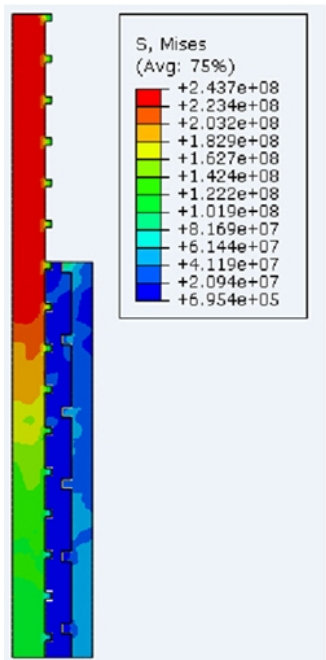

(a)

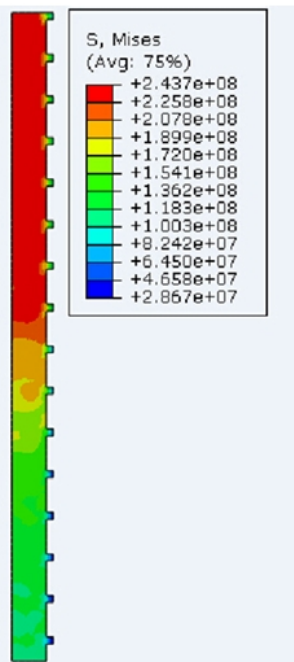

(b)

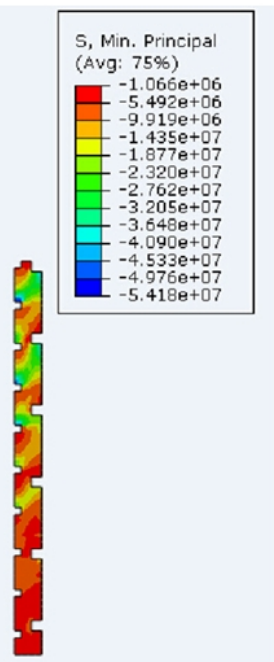

(c)

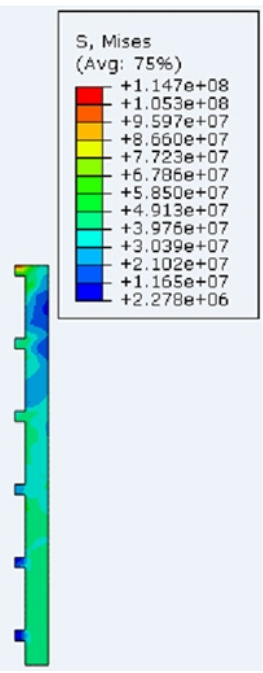

(d)

Fig.3. The stress nephogram of 20GS-2 specimen component

For specimen 20GS-1 and 20GS-2, the grouting-material minimum-principal-stress is compressive, and its extreme value occurs on the surface contacting with reinforcing rib and sleeve rib, of -74 Mpa and -54 Mpa respectively. When component undergoes axial tensile static load, the extreme minimumprincipal-stress and stain at grouting-material presents as non-linear elastic relationship, as shown in Figure4(a). Contrarily, when cyclic tensile load acting on component, the initial curve relating between 
extreme minimum-principal-stress and stain at grouting-material presents non-linear elastic trend, whereas strain does not recover to zero when unloading to $0.02 f_{\mathrm{yk}}$, which implies that residual plastic strain exists leading to the pullout displacement of reinforcement.

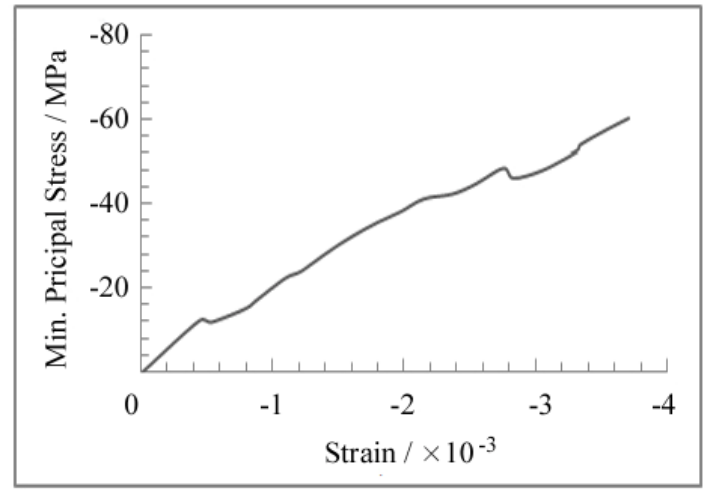

(a) 20GS-1

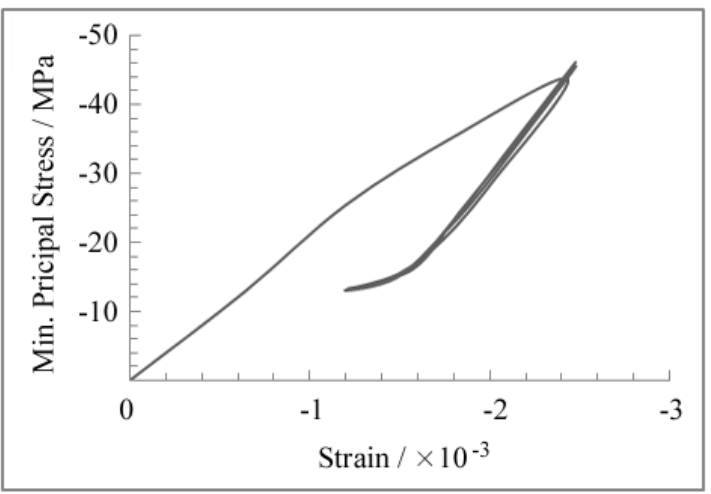

(b) 20GS-2

Fig.4. Stress-stain curve of grouting-material

\section{Conclusion}

In this paper, ABAQUS numerical model of grouting-sleeve reinforcement-connection component was established. Under the action of tension load in accordance with two difference loading system, the stress distribution at component was gotten. Numerical simulation shows conclusions as follows.

(1) Whether axial static load or unidirectional cyclic load imposed on the end of reinforcement, the maximum Mises stress of sleeve occurs at mid-span cross section in axial direction, and the Mises stress at reinforcement bars and sleeve are all in elastic status.

(2) When axial static load imposed on the end of reinforcement, the relationship between extreme minimum-principal-stress and stain at grouting-material presents as non-linear elasticity. However, when cyclic tensile load imposed on the end of reinforcement, strain at grouting-material does not recover to zero when unloading, which implies that residual plastic strain exists, and would lead to the pullout phenomenon of reinforcement from sleeve.

\section{Acknowledgment}

This work was supported by the National Natural Science Foundation of China (No.51278312) and Innovation Team Foundation of Liaoning Education Department (No. LT2012012).

\section{References}

[1] Samuel Yen-Liang Yin. Differential Competition Strategy: R\&D of Ruentex Construction [A]. 4th CECAR Special Forum, 2007: 25-28.

[2] Yang Yang. New Technology of Reinforcement Connection by Grouting-sleeve [J]. Construction Technology, 1995 (05): 45-46, (In Chinese).

[3] Ziliang Wu. Technology of Grouting-sleeve Reinforcement-connection Component[J]. Housing Industry, 2011 (6): 59-61, (In Chinese).

[4] Nobuyuki Izumi, Satoshi Hamada, et al.. Research and Development of Super-high Strength Precast Reinforced Concrete Column [R]. Toda Construction Technical Research Report, No.31, 2002, (In Japanese).

[5] Heng Qin, Guanlong Qian. The Construction Quality Control Measures of Coupler Grouting Splice for Reinforcement [J]. Construction Technology, 2013 (14): 113-117, (In Chinese). 
[6] Hiroshi Tokuda et al. Experimental study of ultra high strength precast reinforced concrete columns [C]. Concrete Institute Proceedings, 2002, 24(2): 727-732, (In Japanese).

[7] Jinhai Liu, Guolu Li, Xiaoyan Hao, et al.. Correlation between Matrix and Tensile Behavior of Ductile Cast Iron [J]. Foundry Technology, 2009, 30(3): 329-332, (In Chinese). 